\title{
Airborne radionuclides and heavy metals in High Arctic terrestrial environment as the indicators of sources and transfers of contamination
}

Edyta Łokas ${ }^{1}$, Agata Zaborska ${ }^{2}$, Ireneusz Sobota ${ }^{3}$, Paweł Gaca ${ }^{4}$, Andrew Milton ${ }^{4}$, Paweł Kocurek ${ }^{5}$, Anna Cwanek ${ }^{1}$

${ }^{1}$ Department of Nuclear Physical Chemistry, Institute of Nuclear Physics Polish Academy of Sciences, Kraków, 31-342, Poland

${ }^{2}$ Marine Chemistry and Biochemistry Department, Institute of Oceanology Polish Academy of Sciences, Sopot, 81-712, Poland

${ }^{3}$ Department of Hydrology and Water Management, Polar Research Centre, Nicholas Copernicus University, Toruń, 87-100, Poland

${ }^{4}$ Ocean and Earth Science, University of Southampton, National Oceanography Centre, European Way, Southampton, SO14 3ZH United Kingdom

${ }^{5}$ Research and Development Laboratory for Aerospace Materials, Rzeszow University of Technology, Rzeszow, 35-959, Poland

Correspondence to: Edyta Łokas (Edyta.Lokas@ifj.edu.pl) 
Tab. S1. Coordinates and selected morphology properties of cryoconite holes on Waldemarbreen.

\begin{tabular}{cccccc}
\hline $\mathbf{N}$ & $\begin{array}{r}\text { GPS coordinates } \\
\text { UTM 33N }(\mathbf{m})\end{array}$ & $\begin{array}{c}\text { Altitude } \\
\text { a.s.l. }(\mathbf{m})\end{array}$ & Area $\left(\mathbf{c m}^{2}\right)$ & Depth $(\mathbf{c m})$ \\
\hline 2 & 435222.3 & 8735590 & 223 & 165 & 29 \\
3 & 435869.9 & 8735874 & 308 & 156 & 10 \\
4 & 436175.5 & 8736001 & 357 & 476 & 28 \\
5 & 435295.8 & 8735921 & 255 & 80 & 9 \\
6 & 435512.6 & 8734906 & 201 & 12.3 & 1 \\
7 & 435954.4 & 8735202 & 233 & 25 & 2.5 \\
8 & 435668.5 & 8735657 & 264 & 121 & 5.5 \\
9 & 434772.3 & 8735363 & 165 & 340 & 4 \\
10 & 434772.3 & 8735363 & 165 & 380 & 7 \\
11 & 435668.5 & 8735657 & 264 & 20 & 8 \\
12 & 436175.5 & 8736001 & 357 & 170 & 7 \\
13 & 436858.6 & 8735885 & 423 & 1200 & 16 \\
\hline
\end{tabular}


Tab. S2. Activity concentrations of anthropogenic radionuclides $\left({ }^{137} \mathrm{Cs}\right.$, Pu isotopes, $\left.{ }^{241} \mathrm{Am}\right)$ expressed in $\mathrm{Bq} \mathrm{kg}^{-1}$ and radionuclide activity ratios and mass ratios in soil profiles. All data were corrected for August 2014, the sampling date.

\begin{tabular}{|c|c|c|c|c|c|c|c|c|c|c|c|c|c|}
\hline Soil & $\begin{array}{c}\text { Depth } \\
\text { (cm) }\end{array}$ & $\begin{array}{c}{ }^{238} \mathbf{P u} \\
(\mathrm{Bq} / \mathrm{kg})\end{array}$ & $\begin{array}{c}{ }^{239+240} \mathbf{P u} \\
(\mathrm{Bq} / \mathrm{kg})\end{array}$ & $\begin{array}{c}{ }^{241} \mathrm{Am} \\
(\mathrm{Bq} / \mathrm{kg})\end{array}$ & $\begin{array}{c}{ }^{137} \mathbf{C s} \\
(\mathbf{B q} / \mathbf{k g})\end{array}$ & ${ }^{238} \mathbf{P u} /{ }^{239+240} \mathbf{P u}$ & ${ }^{241} \mathrm{Am} /{ }^{239+240} \mathrm{Pu}$ & ${ }^{239+240} \mathrm{Pu} /{ }^{137} \mathrm{Cs}$ & $\begin{array}{c}{ }^{238} \mathbf{P u} \\
\left(\mathbf{B q} / \mathbf{m}^{2}\right)\end{array}$ & $\begin{array}{l}{ }^{239+240} \mathbf{P u} \\
\left(\mathrm{Bq} / \mathrm{m}^{2}\right)\end{array}$ & $\begin{array}{l}{ }^{241} \mathrm{Am} \\
\left(\mathrm{Bq} / \mathrm{m}^{2}\right)\end{array}$ & $\begin{array}{c}{ }^{137} \mathbf{C s} \\
\left(\mathbf{B q} / \mathbf{m}^{2}\right)\end{array}$ & $\begin{array}{l}\text { LOI } \\
(\%)\end{array}$ \\
\hline S1-1 & 2 & $<0.03$ & $<0.03$ & $<0.1$ & $31 \pm 4$ & - & - & - & - & - & - & $340 \pm 41$ & 15 \\
\hline S1-2 & 4 & $<0.05$ & $1.07 \pm 0.08$ & $0.59 \pm 0.11$ & $<5$ & - & $0.55 \pm 0.11$ & - & - & $25.7 \pm 1.9$ & $14.2 \pm 2.6$ & $120 \pm 24$ & 8 \\
\hline S1-3 & 6 & $<0.03$ & $0.08 \pm 0.01$ & $<0.1$ & $<5$ & - & - & - & - & $1.8 \pm 0.2$ & - & $44 \pm 22$ & 8 \\
\hline S1-4 & 9 & $<0.07$ & $<0.07$ & $<0.03$ & $<2$ & - & - & - & - & - & - & & 9 \\
\hline S1-5 & 13 & $<0.08$ & $<0.08$ & $<0.08$ & $<2$ & - & - & - & - & - & - & & 8 \\
\hline $\begin{array}{c}\text { Inventory } \\
\left(\mathbf{B q ~ m}^{-2}\right)\end{array}$ & & & & & & & & & - & $28 \pm 2$ & $14 \pm 3$ & $500 \pm 90$ & \\
\hline S3-1 & 2 & $<0.03$ & $0.32 \pm 0.03$ & $0.09 \pm 0.01$ & $8 \pm 2$ & - & $0.28 \pm 0.04$ & \begin{tabular}{|l|}
$0.042 \pm 0.013$ \\
\end{tabular} & - & $6.8 \pm 0.6$ & $1.9 \pm 0.2$ & $148 \pm 42$ & 8 \\
\hline S3-2 & 4.5 & $<0.03$ & $<0.03$ & $<0.03$ & $<4$ & & & - & - & - & - & - & 9 \\
\hline S3-3 & 8 & $<0.03$ & $<0.03$ & $<0.03$ & $<5$ & & & - & - & - & - & - & 8 \\
\hline S3-4 & 12.5 & $<0.03$ & $<0.03$ & $<0.03$ & $<5$ & & & - & - & - & - & - & 10 \\
\hline $\begin{array}{c}\text { Inventory } \\
\left(\mathrm{Bq} \mathrm{m}^{-2}\right)\end{array}$ & & & & & & & & & - & $6.8 \pm 0.6$ & $1.9 \pm 0.2$ & $150 \pm 40$ & \\
\hline S4-1 & 1 & $0.08 \pm 0.01$ & $2.13 \pm 0.16$ & $0.90 \pm 0.06$ & $63 \pm 7$ & $0.038 \pm 0.005$ & $0.39 \pm 0.03$ & \begin{tabular}{|l|}
$0.034 \pm 0.004$ \\
\end{tabular} & $0.58 \pm 0.1$ & $15.6 \pm 1.2$ & $6.1 \pm 0.2$ & $475 \pm 51$ & 7 \\
\hline S4-2 & 4 & $<0.04$ & $0.56 \pm 0.04$ & $0.30 \pm 0.03$ & $16 \pm 7$ & - & $0.48 \pm 0.06$ & $0.034 \pm 0.006$ & $0.35 \pm 0.1$ & $5.0 \pm 0.4$ & $2.4 \pm 0.3$ & $150 \pm 27$ & 8 \\
\hline S4-3 & 8 & $<0.02$ & $0.08 \pm 0.01$ & $<0.03$ & $2 \pm 1$ & - & - & \begin{tabular}{|l|}
$0.041 \pm 0.021$ \\
\end{tabular} & - & $1.9 \pm 0.2$ & & $47 \pm 24$ & 9 \\
\hline S4-4 & 12 & $<0.03$ & $<0.03$ & & $<4$ & - & - & & - & & & - & 9 \\
\hline $\begin{array}{c}\text { Inventory } \\
\left(\mathbf{B q ~ m}^{-2}\right)\end{array}$ & & & & & & & & & $0.94 \pm 0.2$ & $22 \pm 2$ & $\mathbf{9} \pm \mathbf{1}$ & $670 \pm 100$ & \\
\hline S5-1 & 2 & $<0.04$ & $0.51 \pm 0.04$ & $0.25 \pm 0.03$ & $9 \pm 1$ & - & $0.49 \pm 0.07$ & \begin{tabular}{|l|}
$0.059 \pm 0.009$ \\
\end{tabular} & - & $15.6 \pm 1.1$ & $5.0 \pm 0.6$ & $238 \pm 20$ & 8 \\
\hline S5-2 & 4 & $0.03 \pm 0.01$ & $1.04 \pm 0.08$ & $0.47 \pm 0.06$ & $18 \pm 1$ & $\mid 0.029 \pm 0.010$ & $0.45 \pm 0.07$ & \begin{tabular}{|l|}
$0.056 \pm 0.005$ \\
\end{tabular} & $1.2 \pm 0.4$ & $5.0 \pm 0.4$ & $18.6 \pm 2.4$ & $174 \pm 15$ & 9 \\
\hline S5-3 & 7 & $<0.03$ & $<0.03$ & $<0.06$ & $<2$ & - & - & - & - & $1.9 \pm 0.2$ & - & - & 8 \\
\hline S5-4 & 12 & $<0.02$ & $<0.02$ & & $<2$ & - & - & - & - & - & - & - & 9 \\
\hline $\begin{array}{c}\text { Inventory } \\
\left(\mathbf{B q ~ m}^{-2}\right)\end{array}$ & & & & & & & & & $1.2 \pm 0.4$ & $22 \pm 2$ & $24 \pm 3$ & $400 \pm 30$ & \\
\hline S6-1 & 2 & $0.03 \pm 0.01$ & $0.58 \pm 0.05$ & $0.28 \pm 0.03$ & $23 \pm 1$ & \begin{tabular}{|l|}
$0.052 \pm 0.018$ \\
\end{tabular} & $0.045 \pm 0.05$ & $0.025 \pm 0.003$ & - & $15.3 \pm 1.3$ & $6.9 \pm 0.6$ & $449 \pm 26$ & 8 \\
\hline S6-2 & 5 & $<0.03$ & $<0.03$ & $<0.03$ & $<2$ & - & - & - & - & - & - & - & 9 \\
\hline S6-3 & 10 & $<0.02$ & $<0.02$ & $<0.03$ & $<2$ & - & - & - & - & - & - & - & 9 \\
\hline S6-4 & 17 & $<0.06$ & $<0.06$ & $<0.03$ & $<3$ & - & - & - & - & - & - & - & 8 \\
\hline $\begin{array}{c}\text { Inventory } \\
\left(\mathrm{Bq} \mathrm{m}^{-2}\right)\end{array}$ & & & & & & & & & - & $15 \pm 1$ & $7 \pm 1$ & $450 \pm 30$ & \\
\hline
\end{tabular}


Tab. S3. Activity concentrations of natural radionuclides $\left({ }^{210} \mathrm{~Pb},{ }^{234,238} \mathrm{U},{ }^{230,232} \mathrm{Th}\right)$ expressed in $\mathrm{Bq} \mathrm{kg}{ }^{-1}$ and activity ratio of ${ }^{234} \mathrm{U} /{ }^{238} \mathrm{U}$ in soil profiles. Data for ${ }^{210} \mathrm{~Pb}$ were corrected for August 2014, the sampling date.

\begin{tabular}{|c|c|c|c|c|c|c|c|}
\hline soil & Depth $(\mathrm{cm})$ & $\begin{array}{c}{ }^{210} \mathrm{~Pb} \\
(\mathrm{~Bq} / \mathrm{kg})\end{array}$ & $\begin{array}{c}{ }^{234} \mathrm{U} \\
(\mathrm{Bq} / \mathrm{kg})\end{array}$ & $\begin{array}{c}{ }^{238} \mathrm{U} \\
(\mathrm{Bq} / \mathrm{kg})\end{array}$ & ${ }^{234} \mathrm{U} /{ }^{238} \mathrm{U}$ & $\begin{array}{c}{ }^{230} \mathrm{Th} \\
(\mathrm{Bq} / \mathrm{kg})\end{array}$ & $\begin{array}{c}{ }^{232} \mathrm{Th} \\
(\mathrm{Bq} / \mathrm{kg})\end{array}$ \\
\hline S1-1 & 2 & $65 \pm 2$ & $23 \pm 2$ & $22 \pm 2$ & $1.0 \pm 0.1$ & $17 \pm 1$ & $32 \pm 3$ \\
\hline S1-2 & 4 & $43 \pm 1$ & $21 \pm 1$ & $22 \pm 2$ & $0.9 \pm 0.1$ & $22 \pm 2$ & $42 \pm 3$ \\
\hline S1-3 & 6 & $15 \pm 1$ & $22 \pm 2$ & $23 \pm 2$ & $1.0 \pm 0.1$ & $9 \pm 1$ & $8 \pm 1$ \\
\hline S1-4 & 9 & $17 \pm 1$ & $21 \pm 1$ & $22 \pm 1$ & $1.0 \pm 0.1$ & $12 \pm 2$ & $14 \pm 2$ \\
\hline S1-5 & 13 & $15 \pm 1$ & $21 \pm 1$ & $22 \pm 1$ & $1.0 \pm 0.1$ & - & - \\
\hline S3-1 & 2 & $37 \pm 5$ & $16 \pm 1$ & $16 \pm 1$ & $1.0 \pm 0.1$ & $9 \pm 1$ & $17 \pm 2$ \\
\hline S3-2 & 4.5 & $15 \pm 4$ & $16 \pm 1$ & $15 \pm 1$ & $1.1 \pm 0.1$ & $10 \pm 1$ & $18 \pm 2$ \\
\hline S3-3 & 8 & $17 \pm 4$ & $15 \pm 1$ & $14 \pm 1$ & $1.0 \pm 0.1$ & $11 \pm 1$ & $20 \pm 2$ \\
\hline S3-4 & 12.5 & $16 \pm 4$ & $17 \pm 1$ & $14 \pm 1$ & $1.2 \pm 0.1$ & $10 \pm 1$ & $19 \pm 1$ \\
\hline S4-1 & 1 & $173 \pm 4$ & $21 \pm 1$ & $20 \pm 1$ & $1.1 \pm 0.1$ & $10 \pm 1$ & $21 \pm 2$ \\
\hline S4-2 & 4 & $31 \pm 1$ & $20 \pm 1$ & $18 \pm 1$ & $1.1 \pm 0.2$ & $10 \pm 1$ & $27 \pm 2$ \\
\hline S4-3 & 8 & $29 \pm 2$ & $19 \pm 1$ & $18 \pm 1$ & $1.1 \pm 0.1$ & $11 \pm 1$ & $29 \pm 2$ \\
\hline S4-4 & 12 & $27 \pm 3$ & $21 \pm 1$ & $20 \pm 1$ & $1.0 \pm 0.1$ & $10 \pm 1$ & $29 \pm 2$ \\
\hline S5-1 & 2 & $83 \pm 7$ & $23 \pm 2$ & $21 \pm 1$ & $1.1 \pm 0.1$ & $11 \pm 1$ & $19 \pm 1$ \\
\hline S5-2 & 4 & $57 \pm 8$ & $28 \pm 2$ & $30 \pm 2$ & $0.9 \pm 0.1$ & $15 \pm 1$ & $34 \pm 3$ \\
\hline S5-3 & 7 & $21 \pm 2$ & $17 \pm 1$ & $17 \pm 1$ & $1.0 \pm 0.1$ & $10 \pm 1$ & $15 \pm 1$ \\
\hline S5-4 & 12 & $18 \pm 2$ & $15 \pm 1$ & $16 \pm 1$ & $1.0 \pm 0.1$ & $10 \pm 1$ & $20 \pm 2$ \\
\hline S6-1 & 2 & $82 \pm 6$ & $24 \pm 2$ & $22 \pm 2$ & $1.1 \pm 0.1$ & $11 \pm 1$ & $16 \pm 1$ \\
\hline S6-2 & 5 & $21 \pm 4$ & $14 \pm 1$ & $13 \pm 1$ & $1.0 \pm 0.1$ & $10 \pm 1$ & $17 \pm 1$ \\
\hline S6-3 & 10 & $18 \pm 2$ & $19 \pm 1$ & $19 \pm 1$ & $1.0 \pm 0.1$ & $10 \pm 1$ & $21 \pm 2$ \\
\hline S6-4 & 17 & $19 \pm 2$ & $13 \pm 1$ & $12 \pm 1$ & $1.1 \pm 0.1$ & $9 \pm 1$ & $13 \pm 1$ \\
\hline
\end{tabular}


Tab. S4. Activity concentrations of anthropogenic radionuclides $\left({ }^{137} \mathrm{Cs}\right.$, Pu isotopes, $\left.{ }^{241} \mathrm{Am}\right)$ expressed in $\mathrm{Bq} \mathrm{kg}^{-1}$ and radionuclide activity ratios and mass ratios in all cryoconite samples. All data were corrected for August 2014, the sampling date.

\begin{tabular}{|c|c|c|c|c|c|c|c|c|}
\hline No. & $\begin{array}{c}{ }^{137} \mathrm{Cs} \\
(\mathrm{Bq} / \mathrm{kg})\end{array}$ & $\begin{array}{c}{ }^{239+240} \mathrm{Pu} \\
(\mathrm{Bq} / \mathrm{kg})\end{array}$ & $\begin{array}{c}{ }^{238} \mathrm{Pu} \\
(\mathrm{Bq} / \mathrm{kg})\end{array}$ & $\begin{array}{c}{ }^{241} \mathrm{Am} \\
(\mathrm{Bq} / \mathrm{kg})\end{array}$ & ${ }^{238} \mathrm{Pu} /{ }^{239 \pm 240} \mathrm{Pu}$ & ${ }^{239 \pm 240} \mathrm{Pu} /{ }^{137} \mathrm{Cs}$ & ${ }^{241} \mathrm{Am} /{ }^{239 \pm 240} \mathrm{Pu}$ & ${ }^{240} \mathrm{Pu}{ }^{239} \mathrm{Pu}$ \\
\hline KW2 & $642 \pm 84$ & $16.73 \pm 1.12$ & $0.80 \pm 0.10$ & $7.56 \pm 0.54$ & $0.048 \pm 0.007$ & $0.026 \pm 0.004$ & $0.45 \pm 0.04$ & $0.159 \pm 0.002$ \\
\hline KW3 & $1021 \pm 136$ & $16.93 \pm 1.15$ & $0.59 \pm 0.08$ & $7.52 \pm 0.57$ & $0.035 \pm 0.005$ & $0.017 \pm 0.002$ & $0.44 \pm 0.05$ & $0.145 \pm 0.001$ \\
\hline KW4 & $2030 \pm 257$ & $33.54 \pm 2.30$ & $2.09 \pm 0.22$ & $18.77 \pm 1.27$ & $0.062 \pm 0.008$ & $0.017 \pm 0.002$ & $0.56 \pm 0.05$ & $0.141 \pm 0.001$ \\
\hline KW5 & $109 \pm 22$ & $1.59 \pm 0.16$ & $0.08 \pm 0.02$ & $1.04 \pm 0.16$ & $0.050 \pm 0.013$ & $0.015 \pm 0.003$ & $0.65 \pm 0.12$ & $0.196 \pm 0.019$ \\
\hline KW6 & $<3$ & $0.09 \pm 0.02$ & $<0.03$ & $<0.25$ & - & - & - & - \\
\hline KW7 & $17 \pm 7$ & $0.12 \pm 0.02$ & $<0.02$ & $<0.20$ & - & $0.007 \pm 0.003$ & - & - \\
\hline KW8 & $440 \pm 58$ & $7.20 \pm 0.57$ & $0.45 \pm 0.07$ & $3.02 \pm 0.26$ & $0.063 \pm 0.011$ & $0.016 \pm 0.003$ & $0.42 \pm 0.05$ & $0.161 \pm 0.004$ \\
\hline KW9 & $13 \pm 3$ & $0.47 \pm 0.07$ & $<0.04$ & $0.25 \pm 0.06$ & - & $0.035 \pm 0.010$ & $0.53 \pm 0.16$ & $0.121 \pm 0.039$ \\
\hline KW10 & $76 \pm 13$ & $2.88 \pm 0.27$ & $0.15 \pm 0.06$ & $1.45 \pm 0.20$ & $0.052 \pm 0.022$ & $0.038 \pm 0.008$ & $0.50 \pm 0.08$ & $0.131 \pm 0.015$ \\
\hline KW11 & $513 \pm 88$ & $7.40 \pm 0.54$ & $0.23 \pm 0.04$ & $1.80 \pm 0.36$ & $0.031 \pm 0.006$ & $0.014 \pm 0.003$ & $0.24 \pm 0.05$ & $0.137 \pm 0.004$ \\
\hline KW12 & $1886 \pm 264$ & $33.64 \pm 2.33$ & $2.08 \pm 0.23$ & $18.13 \pm 1.24$ & $0.062 \pm 0.008$ & $0.018 \pm 0.003$ & $0.54 \pm 0.05$ & $0.140 \pm 0.001$ \\
\hline KW13 & $1905 \pm 266$ & $42.77 \pm 2.81$ & $2.10 \pm 0.20$ & $24.48 \pm 1.47$ & $0.049 \pm 0.006$ & $0.022 \pm 0.003$ & $0.57 \pm 0.05$ & $0.143 \pm 0.001$ \\
\hline
\end{tabular}

Tab. S5. Activity concentrations of natural radionuclides $\left({ }^{210} \mathrm{~Pb},{ }^{234,238} \mathrm{U},{ }^{230,232} \mathrm{Th}\right)$ expressed in $\mathrm{Bq} \mathrm{kg}{ }^{-1}$ and activity ratio of ${ }^{234} \mathrm{U} /{ }^{238} \mathrm{U}$ in all cryoconite samples. Data for ${ }^{210} \mathrm{~Pb}$ were corrected for August 2014, the sampling date.

\begin{tabular}{|c|c|c|c|c|c|c|c|}
\hline No. & $\begin{array}{c}{ }^{210} \mathrm{~Pb} \\
(\mathrm{~Bq} / \mathrm{kg})\end{array}$ & $\begin{array}{c}{ }^{234} \mathrm{U} \\
(\mathrm{Bq} / \mathrm{kg})\end{array}$ & $\begin{array}{c}{ }^{238} \mathrm{U} \\
(\mathrm{Bq} / \mathrm{kg})\end{array}$ & ${ }^{234} \mathrm{U} /{ }^{238} \mathrm{U}$ & $\begin{array}{c}{ }^{230} \mathrm{Th} \\
(\mathrm{Bq} / \mathrm{kg})\end{array}$ & $\begin{array}{c}{ }^{232} \mathrm{Th} \\
(\mathrm{Bq} / \mathrm{kg})\end{array}$ & LOI (\%) \\
\hline KW2 & $3577 \pm 196$ & $18 \pm 2$ & $18 \pm 2$ & $1.0 \pm 0.2$ & $18 \pm 1$ & $16 \pm 1$ & 6 \\
\hline KW3 & $4460 \pm 230$ & $43 \pm 4$ & $37 \pm 4$ & $1.2 \pm 0.2$ & $54 \pm 4$ & $50 \pm 4$ & 6 \\
\hline KW4 & $12269 \pm 634$ & $34 \pm 3$ & $36 \pm 4$ & $0.9 \pm 0.1$ & $45 \pm 3$ & $48 \pm 4$ & 11 \\
\hline KW5 & $1028 \pm 73$ & $25 \pm 2$ & $25 \pm 3$ & $1.0 \pm 0.1$ & $33 \pm 2$ & $57 \pm 4$ & 2 \\
\hline KW6 & $485 \pm 56$ & $16 \pm 2$ & $12 \pm 2$ & $1.4 \pm 0.3$ & $25 \pm 2$ & $56 \pm 4$ & 3 \\
\hline KW7 & $497 \pm 35$ & $12 \pm 1$ & $10 \pm 1$ & $1.2 \pm 0.2$ & $16 \pm 1$ & $35 \pm 3$ & 3 \\
\hline KW8 & $4443 \pm 224$ & $22 \pm 2$ & $23 \pm 2$ & $1.0 \pm 0.1$ & $27 \pm 2$ & $51 \pm 4$ & 8 \\
\hline KW9 & $1744 \pm 95$ & $26 \pm 2$ & $24 \pm 2$ & $1.1 \pm 0.1$ & $27 \pm 2$ & $58 \pm 4$ & 6 \\
\hline KW10 & $2739 \pm 142$ & $20 \pm 2$ & $20 \pm 2$ & $1.0 \pm 0.1$ & $27 \pm 2$ & $54 \pm 4$ & 7 \\
\hline
\end{tabular}




\begin{tabular}{|l|l|l|l|l|l|l|l|}
\hline KW11 & $5053 \pm 292$ & $23 \pm 2$ & $24 \pm 2$ & $1.0 \pm 0.1$ & $34 \pm 2$ & $50 \pm 4$ & 8 \\
\hline KW12 & $5081 \pm 259$ & $39 \pm 3$ & $31 \pm 3$ & $1.3 \pm 0.2$ & $47 \pm 4$ & $47 \pm 4$ & 11 \\
\hline KW13 & $5236 \pm 263$ & $36 \pm 3$ & $33 \pm 2$ & $1.1 \pm 0.1$ & $41 \pm 3$ & $48 \pm 4$ & 11 \\
\hline
\end{tabular}

Table S6. Concentrations of measured metals in soil samples, together with ${ }^{206} \mathrm{~Pb}^{1207} \mathrm{~Pb}^{\text {and }}{ }^{208} \mathrm{~Pb}^{1206} \mathrm{~Pb}^{12}$ ratios. The calculated anthropogenic metal

enrichment factors (EF) results are also given after normalization for Fe and Al content (Fe norm or $\mathrm{Al} \mathrm{norm}$ ).

\begin{tabular}{|c|c|c|c|c|c|c|c|c|c|c|c|c|c|c|c|c|c|c|c|c|}
\hline & $\begin{array}{c}\mathbf{P b} \\
(\mathbf{m g} / \mathbf{k g})\end{array}$ & $\begin{array}{c}\mathbf{Z n} \\
(\mathbf{m g} / \mathbf{k g})\end{array}$ & $\begin{array}{c}\mathbf{C u} \\
(\mathbf{m g} / \mathbf{k g})\end{array}$ & $\begin{array}{c}\mathbf{C d} \\
(\mathbf{m g} / \mathbf{k g})\end{array}$ & $\begin{array}{c}\mathbf{F e} \\
(\mathrm{g} / \mathbf{k g})\end{array}$ & $\begin{array}{c}\mathrm{Al} \\
(\mathrm{g} / \mathbf{k g})\end{array}$ & $\begin{array}{c}\mathbf{C r} \\
(\mathbf{m g} / \mathbf{k g})\end{array}$ & $\begin{array}{c}\text { Co } \\
(\mathbf{m g} / \mathbf{k g})\end{array}$ & $\begin{array}{c}\mathbf{N i} \\
(\mathbf{m g} / \mathbf{k g})\end{array}$ & $\begin{array}{c}\mathrm{Mn} \\
(\mathbf{m g} / \mathbf{k g})\end{array}$ & $\begin{array}{l}{ }^{206} \mathrm{~Pb} / \\
{ }^{207} \mathrm{~Pb}\end{array}$ & $\begin{array}{l}{ }^{208} \mathrm{~Pb} / \\
{ }^{206} \mathrm{~Pb}\end{array}$ & $\begin{array}{l}\text { EF Pb } \\
\text { Fe norm } \\
\end{array}$ & $\begin{array}{l}\mathbf{E F} \\
\mathbf{P b} \\
\text { Al norm }\end{array}$ & $\begin{array}{l}\text { EF Zn } \\
\text { Fe norm } \\
\end{array}$ & $\begin{array}{l}\text { EF Zn } \\
\text { Al norm } \\
\end{array}$ & $\begin{array}{l}\text { EF Cu } \\
\text { Fe norm } \\
\end{array}$ & $\begin{array}{l}\text { EF Cu } \\
\text { Al norm } \\
\end{array}$ & $\begin{array}{l}\text { EF Cd } \\
\text { Fe norm } \\
\end{array}$ & $\begin{array}{l}\text { EF Cd } \\
\text { Al norm } \\
\end{array}$ \\
\hline SO1-1 & 20.75 & 66.56 & 22.93 & 0.12 & 38.02 & 51.79 & 55.26 & 11.12 & 30.25 & 0.67 & 1.205 & 2.046 & 1.6 & 1.3 & 1.0 & 0.9 & 1.4 & 1.2 & 1.1 & 0.7 \\
\hline SO1-2 & 21.13 & 75.26 & 26.57 & 0.05 & 42.91 & 64.47 & 61.20 & 12.37 & 32.22 & 0.83 & 1.205 & 2.035 & 1.4 & 1.1 & 1.0 & 0.8 & 1.4 & 1.1 & 0.4 & 0.4 \\
\hline SO1-3 & 20.94 & 71.30 & 25.95 & 0.09 & 44.18 & 62.30 & 52.20 & 12.46 & 30.63 & 0.78 & 1.205 & 2.036 & 1.4 & 1.1 & 0.9 & 0.8 & 1.4 & 1.1 & 0.7 & 0.4 \\
\hline SO1-4 & 19.19 & 65.67 & 23.88 & 0.60 & 39.95 & 58.99 & 53.61 & 11.74 & 28.16 & 0.87 & 1.203 & 2.048 & 1.4 & 1.1 & 1.0 & 0.7 & 1.4 & 1.1 & 5.3 & 3.1 \\
\hline SO1-5 & 18.92 & 66.04 & 24.55 & 0.07 & 39.72 & 62.58 & 50.27 & 11.60 & 28.61 & 0.72 & 1.208 & 2.036 & 1.4 & 1.0 & 1.0 & 0.7 & 1.4 & 1.0 & 0.6 & 0.4 \\
\hline SO5-1 & 14.73 & 58.41 & 16.90 & 0.10 & 26.18 & 47.41 & 33.24 & 8.86 & 60.27 & 0.49 & 1.206 & 2.036 & 1.6 & 1.0 & 1.3 & 0.8 & 1.5 & 1.0 & 1.3 & 0.6 \\
\hline SO5-3 & 9.59 & 45.80 & 12.24 & 0.27 & 27.46 & 37.19 & 28.85 & 6.13 & 18.70 & 0.39 & 1.211 & 2.039 & 1.0 & 0.9 & 1.0 & 0.8 & 1.0 & 0.9 & 3.4 & 2.2 \\
\hline SO5-4 & 20.24 & 62.94 & 20.61 & 0.36 & 32.45 & 54.91 & 46.49 & 10.55 & 27.31 & 0.38 & 1.208 & 2.040 & 1.8 & 1.2 & 1.1 & 0.8 & 1.5 & 1.0 & 3.9 & 2.0 \\
\hline SO6-1 & 10.07 & 48.05 & 12.95 & 0.21 & 35.62 & 42.22 & 32.47 & 7.23 & 23.86 & 0.35 & 1.209 & 2.030 & 0.8 & 0.8 & 0.8 & 0.8 & 0.8 & 0.8 & 2.1 & 1.5 \\
\hline SO6-2 & 12.70 & 47.45 & 14.71 & 0.60 & 27.41 & 41.18 & 34.42 & 6.98 & 24.13 & 0.34 & 1.197 & 2.057 & 1.4 & 1.0 & 1.0 & 0.8 & 1.3 & 1.0 & 7.7 & 4.4 \\
\hline SO6-3 & 15.51 & 50.78 & 26.18 & 0.50 & 28.58 & 38.52 & 46.85 & 10.67 & 28.86 & 0.52 & 1.217 & 2.050 & 1.6 & 1.3 & 1.0 & 0.9 & 2.1 & 1.8 & 6.2 & 3.9 \\
\hline SO6-4 & 15.80 & 53.17 & 22.52 & 0.60 & 28.87 & 43.24 & 42.65 & 10.87 & 28.41 & 0.57 & 1.208 & 2.042 & 1.6 & 1.2 & 1.1 & 0.8 & 1.8 & 1.4 & 7.3 & 4.2 \\
\hline SO4-1 & 25.91 & 76.89 & 19.59 & 0.34 & 28.72 & 47.41 & 38.47 & 8.53 & 21.83 & 0.40 & 1.190 & 2.027 & 2.6 & 1.8 & 1.6 & 1.1 & 1.6 & 1.1 & 4.2 & 2.2 \\
\hline SO4-3 & 17.14 & 53.07 & 20.73 & 0.14 & 28.91 & 59.96 & 62.65 & 10.86 & 26.23 & 0.46 & 1.196 & 2.036 & 1.7 & 1.0 & 1.1 & 0.6 & 1.7 & 0.9 & 1.6 & 0.7 \\
\hline SO4-4 & 19.36 & 53.42 & 22.40 & 0.16 & 34.00 & 58.64 & 30.21 & 11.58 & 29.12 & 0.48 & 1.203 & 2.033 & 1.7 & 1.1 & 0.9 & 0.6 & 1.5 & 1.0 & 1.6 & 0.8 \\
\hline SO3-1 & 11.57 & 45.09 & 11.83 & 0.22 & 20.85 & 40.20 & 36.12 & 5.37 & 15.01 & 0.35 & 1.190 & 2.025 & 1.6 & 1.0 & 1.3 & 0.7 & 1.3 & 0.8 & 3.6 & 1.6 \\
\hline SO3-2 & 11.34 & 51.80 & 12.92 & 0.17 & 30.56 & 43.56 & 37.21 & 6.94 & 18.46 & 0.53 & 1.197 & 2.038 & 1.1 & 0.9 & 1.0 & 0.8 & 1.0 & 0.8 & 2.0 & 1.2 \\
\hline SO3-3 & 12.91 & 59.38 & 14.37 & 0.15 & 30.32 & 38.73 & 33.39 & 6.79 & 20.99 & 0.57 & 1.200 & 2.014 & 1.2 & 1.1 & 1.1 & 1.0 & 1.1 & 1.0 & 1.8 & 1.2 \\
\hline SO3-4 & 12.82 & 56.62 & 14.42 & 0.18 & 27.89 & 45.30 & 30.11 & 7.26 & 20.32 & 0.74 & 1.204 & 2.022 & 1.3 & 0.9 & 1.2 & 0.8 & 1.2 & 0.8 & 2.2 & 1.2 \\
\hline
\end{tabular}


Table S7. The Pearson's correlation matrix for soil samples. Different heavy metals concentrations, ${ }^{206} \mathrm{~Pb} /{ }^{207} \mathrm{~Pb}$ and ${ }^{208} \mathrm{~Pb} /{ }^{206} \mathrm{~Pb}$ isotopic ratios, natural and anthropogenic radionuclide activity concentrations and organic matter content (LOI).

\begin{tabular}{|c|c|c|c|c|c|c|c|c|c|c|c|c|c|c|c|c|c|c|c|c|}
\hline Soils & ${ }^{234} \mathrm{U}$ & ${ }^{238} \mathbf{U}$ & ${ }^{230} \mathrm{Th}$ & ${ }^{{ }^{232} \mathrm{Th}}$ & $\mathrm{Cr}$ & $\mathbf{P b}$ & $\mathrm{Cu}$ & $\mathrm{Zn}$ & Cd & $\begin{array}{l}\text { Co } \\
\end{array}$ & $\mathrm{Ni}$ & Mn & $\mathrm{Fe}$ & Al & ${ }^{20661207} \mathbf{P b}$ & ${ }^{20882106} \mathbf{P b}$ & LOI & ${ }^{137} \mathrm{Cs}$ & ${ }^{239+241} \mathbf{P u}$ & ${ }^{241} \mathbf{A m}$ \\
\hline${ }^{210} \mathrm{~Pb}$ & 0.65 & 0.68 & 0.80 & 0.55 & 0.63 & 0.74 & 0.53 & 0.71 & 0.05 & 0.45 & 0.40 & $\begin{array}{l}-0.39 \\
\end{array}$ & 0.41 & 0.51 & $\begin{array}{l}-0.70 \\
\end{array}$ & 0.03 & 0.32 & 0.90 & 0.79 & 0.71 \\
\hline${ }^{234} \mathrm{U}$ & & 0.95 & 0.83 & 0.53 & 0.48 & 0.42 & 0.31 & 0.60 & -0.22 & 0.34 & 0.42 & -0.14 & 0.32 & 0.36 & -0.57 & 0.31 & 0.35 & 0.20 & 0.20 & 0.28 \\
\hline${ }^{238} \mathrm{U}$ & & & 0.77 & 0.50 & 0.46 & 0.42 & 0.31 & 0.57 & -0.20 & 0.32 & 0.39 & -0.12 & 0.32 & 0.35 & -0.53 & 0.29 & 0.36 & 0.08 & 0.21 & 0.32 \\
\hline${ }^{230} \mathrm{Th}$ & & & & 0.80 & 0.75 & 0.63 & 0.57 & 0.82 & -0.05 & 0.60 & 0.45 & -0.25 & 0.56 & 0.64 & -0.69 & 0.22 & 0.26 & -0.09 & 0.13 & 0.31 \\
\hline${ }^{232} \mathrm{Th}$ & & & & & 0.76 & 0.50 & 0.64 & 0.74 & -0.16 & 0.73 & 0.36 & -0.23 & 0.58 & 0.78 & -0.48 & 0.26 & 0.19 & 0.00 & 0.22 & 0.39 \\
\hline $\mathrm{Cr}$ & & & & & & 0.73 & 0.86 & 0.88 & 0.01 & 0.88 & 0.51 & -0.15 & 0.80 & 0.89 & -0.62 & 0.26 & 0.17 & -0.33 & -0.28 & 0.29 \\
\hline $\mathbf{P b}$ & & & & & & & 0.82 & 0.71 & 0.09 & 0.59 & 0.48 & -0.40 & 0.53 & 0.59 & -0.78 & -0.08 & 0.25 & 0.46 & 0.47 & 0.90 \\
\hline $\mathrm{Cu}$ & & & & & & & & 0.77 & 0.11 & 0.89 & 0.56 & -0.12 & 0.73 & 0.80 & -0.59 & -0.01 & 0.07 & -0.12 & -0.04 & 0.56 \\
\hline Zn & & & & & & & & & -0.01 & 0.80 & 0.56 & -0.08 & 0.75 & 0.84 & -0.59 & 0.21 & 0.30 & 0.35 & 0.41 & 0.83 \\
\hline Cd & & & & & & & & & & 0.04 & -0.03 & -0.08 & -0.04 & -0.10 & -0.03 & -0.25 & -0.11 & 0.74 & 0.51 & 0.30 \\
\hline Co & & & & & & & & & & & 0.57 & 0.04 & 0.84 & 0.91 & -0.43 & 0.19 & -0.04 & -0.24 & \begin{tabular}{c|}
-0.18 \\
\end{tabular} & 0.42 \\
\hline $\mathrm{Ni}$ & & & & & & & & & & & & -0.04 & 0.49 & 0.48 & -0.54 & -0.02 & -0.01 & -0.24 & -0.16 & -0.12 \\
\hline Mn & & & & & & & & & & & & & 0.14 & -0.09 & 0.51 & 0.09 & -0.25 & -0.30 & -0.17 & 0.34 \\
\hline $\mathrm{Fe}$ & & & & & & & & & & & & & & 0.82 & -0.45 & 0.19 & -0.12 & -0.42 & -0.42 & -0.03 \\
\hline Al & & & & & & & & & & & & & & & -0.50 & 0.31 & 0.05 & -0.40 & -0.16 & 0.42 \\
\hline${ }^{2066 / 2107} \mathbf{P b}$ & & & & & & & & & & & & & & & & 0.06 & -0.12 & 0.04 & -0.02 & 0.49 \\
\hline${ }^{2088201060} \mathbf{P b}$ & & & & & & & & & & & & & & & & & 0.12 & $\begin{array}{c}.0 .19 \\
\end{array}$ & -0.26 & -0.08 \\
\hline LOI & & & & & & & & & & & & & & & & & & 0.70 & 0.45 & 0.34 \\
\hline $\begin{array}{ll}137 \mathrm{Cs} \\
\end{array}$ & & & & & & & & & & & & & & & & & & & 0.72 & 0.74 \\
\hline $\begin{array}{l}2399+240 \mathrm{Pu} \\
\end{array}$ & & & & & & & & & & & & & & & & & & & & 0.98 \\
\hline
\end{tabular}


Table S8. Concentrations of measured metals in cryoconite samples, together with ${ }^{206} \mathrm{~Pb}^{1207} \mathrm{~Pb}$ and ${ }^{208} \mathrm{~Pb}^{1206} \mathrm{~Pb}$ ratios. The calculated anthropogenic metal enrichment factors (EF) results are also given after normalization for Fe and Al content (Fe norm or ${ }_{\mathrm{Al}}$ norm).

\begin{tabular}{|c|c|c|c|c|c|c|c|c|c|c|c|c|c|c|c|c|c|c|c|c|}
\hline & $\begin{array}{c}\mathrm{Pb} \\
(\mathrm{mg} / \mathrm{kg})\end{array}$ & $\begin{array}{c}\mathrm{Zn} \\
(\mathbf{m g} / \mathbf{k g})\end{array}$ & $\underset{(\mathrm{mg} / \mathrm{kg})}{\mathrm{Cu}}$ & $\underset{(\mathrm{mg} / \mathrm{kg})}{\mathrm{Cd}}$ & $\begin{array}{c}\mathbf{F e} \\
(\mathrm{g} / \mathrm{kg})\end{array}$ & $\underset{(\mathrm{g} / \mathrm{kg})}{\mathrm{Al}}$ & $\underset{(\mathrm{mg} / \mathrm{kg})}{\mathrm{Cr}}$ & $\underset{(\mathrm{mg} / \mathrm{kg})}{\mathrm{Co}}$ & $\begin{array}{c}\mathrm{Ni} \\
(\mathrm{mg} / \mathrm{kg})\end{array}$ & $\underset{(\mathbf{m g} / \mathbf{k g})}{\mathrm{Mn}}$ & $\begin{array}{l}{ }^{206} \mathrm{~Pb} / \\
{ }^{207} \mathrm{~Pb}\end{array}$ & $\begin{array}{l}{ }^{208} \mathrm{~Pb} / \\
{ }^{206} \mathrm{~Pb}\end{array}$ & $\begin{array}{l}\text { EF Pb } \\
\text { Fe norm }\end{array}$ & $\begin{array}{l}\mathbf{E F} \\
\mathbf{P b} \\
\text { Al norm }\end{array}$ & $\begin{array}{l}\text { EF Zn } \\
\text { Fe norm }\end{array}$ & $\begin{array}{l}\text { EF Zn } \\
\text { Al norm }\end{array}$ & $\begin{array}{l}\text { EF Cu } \\
\text { Fe norm } \\
\end{array}$ & $\begin{array}{l}\text { EF Cu } \\
\text { Al norm }\end{array}$ & $\begin{array}{l}\text { EF Cd } \\
\text { Fe norm } \\
\end{array}$ & $\begin{array}{l}\text { EF Cd } \\
\text { Al norm }\end{array}$ \\
\hline 2 & 82.79 & 85.07 & 34.3 & 0.21 & 49.62 & 83.03 & 83.01 & 13.39 & 37.19 & 0.34 & 1.174 & 2.042 & 5.0 & 3.3 & 1.0 & 0.7 & 1.7 & 1.1 & 1.5 & 0.7 \\
\hline 3 & 23.04 & 96.89 & 22.4 & 0.23 & 42.56 & 75.50 & 77.22 & 13.16 & 35.89 & 0.51 & 1.183 & 2.061 & 1.6 & 1.0 & 1.4 & 0.9 & 1.3 & 0.8 & 1.9 & 0.9 \\
\hline 4 & 95.53 & 92.36 & 34.8 & 0.24 & 37.08 & 69.40 & 75.57 & 13.01 & 36.70 & 0.30 & 1.174 & 2.025 & 7.7 & 4.6 & 1.5 & 0.9 & 2.3 & 1.3 & 2.4 & 1.1 \\
\hline 5 & 40.91 & 74.22 & 27.5 & 0.20 & 42.75 & 77.55 & 64.01 & 13.73 & 29.15 & 0.39 & 1.192 & 2.035 & 2.9 & 1.8 & 1.0 & 0.6 & 1.5 & 0.9 & 1.7 & 0.8 \\
\hline 6 & 36.84 & 87.32 & 33.4 & 0.27 & 51.27 & 89.73 & 80.95 & 17.74 & 34.71 & 0.63 & 1.199 & 2.034 & 2.2 & 1.4 & 1.0 & 0.6 & 1.6 & 1.0 & 1.9 & 0.9 \\
\hline 7 & 24.02 & 59.57 & 25.8 & 0.24 & 29.38 & 63.57 & 47.55 & 11.42 & 22.56 & 0.40 & 1.197 & 2.036 & 2.5 & 1.3 & 1.2 & 0.6 & 2.1 & 1.1 & 2.9 & 1.1 \\
\hline 9 & 57.91 & 90.04 & 40.1 & 0.21 & 41.38 & 91.87 & 83.22 & 15.21 & 36.70 & 0.51 & 1.181 & 2.039 & 4.2 & 2.1 & 1.3 & 0.7 & 2.3 & 1.2 & 1.8 & 0.7 \\
\hline 10 & 45.57 & 83.26 & 34.4 & 0.19 & 46.65 & 79.24 & 81.29 & 14.15 & 33.73 & 0.25 & 1.184 & 2.039 & 2.9 & 1.9 & 1.1 & 0.7 & 1.8 & 1.2 & 1.4 & 0.7 \\
\hline 11 & 97.28 & 97.55 & 39.6 & 0.60 & 48.16 & 78.80 & 84.04 & 15.31 & 41.80 & 0.31 & 1.180 & 2.038 & 6.1 & 4.1 & 1.2 & 0.8 & 2.0 & 1.3 & 4.5 & 2.3 \\
\hline 12 & 97.70 & 86.80 & 34.3 & 0.19 & 37.93 & 65.72 & 76.42 & 12.95 & 36.46 & 0.31 & 1.169 & 2.032 & 7.7 & 5.0 & 1.4 & 0.9 & 2.2 & 1.4 & 1.8 & 0.9 \\
\hline 13 & 19.87 & 88.14 & 21.5 & 0.26 & 40.18 & 85.90 & 65.98 & 13.00 & 35.83 & 0.46 & 1.194 & 2.047 & 1.5 & 0.8 & 1.3 & 0.7 & 1.3 & 0.7 & 2.3 & 0.9 \\
\hline
\end{tabular}


Table S9. The Pearson's correlation matrix for cryoconite samples. Different heavy metals concentrations, ${ }^{206} \mathrm{~Pb} /{ }^{207} \mathrm{~Pb}$ and ${ }^{208} \mathrm{~Pb} /{ }^{206} \mathrm{~Pb}$ isotopic ratios, natural and anthropogenic radionuclide activity concentrations, organic matter content (LOI), and some cryoconite characteristics: localization altitude, area and depth were taken into account.

\begin{tabular}{|c|c|c|c|c|c|c|c|c|c|c|c|c|c|c|c|c|c|c|c|c|c|c|c|c|}
\hline Cryoconites & ${ }^{234} \mathrm{U}$ & ${ }^{238} \mathrm{U}$ & ${ }^{230} \mathrm{Th}$ & ${ }^{{ }^{232} \mathrm{Th}}$ & $\mathrm{Cr}$ & $\mathbf{P b}$ & $\mathrm{Cu}$ & $\mathbf{Z n}$ & Cd & Co & $\mathrm{Ni}$ & Mn & $\mathbf{F e}$ & Al & ${ }^{2066 / 2107} \mathbf{P b}$ & ${ }^{2088206} \mathbf{P b}$ & LOI & ${ }^{137} \mathrm{Cs}$ & ${ }^{239+24+24} \mathbf{P u}$ & ${ }^{241} \mathrm{Am}$ & ${ }^{238} \mathrm{Pu}$ & Altitude & Area & Depth \\
\hline${ }^{210} \mathrm{~Pb}$ & 0.56 & 0.72 & 0.59 & -0.06 & 0.21 & 0.50 & 0.08 & 0.53 & 0.15 & -0.29 & 0.41 & -0.41 & -0.18 & -0.34 & -0.55 & -0.19 & 0.77 & 0.80 & 0.72 & 0.70 & 0.75 & 0.64 & 0.38 & 0.68 \\
\hline${ }^{234} \mathrm{U}$ & & 0.94 & 0.96 & 0.25 & 0.19 & 0.13 & -0.22 & 0.57 & -0.12 & -0.32 & 0.40 & -0.02 & -0.19 & -0.40 & -0.44 & 0.31 & 0.60 & 0.77 & 0.72 & 0.70 & 0.67 & 0.72 & 0.43 & 0.24 \\
\hline${ }^{238} \mathbf{U}$ & & & 0.92 & 0.26 & 0.19 & 0.20 & -0.18 & 0.60 & -0.03 & -0.34 & 0.41 & -0.16 & -0.18 & -0.36 & -0.46 & 0.22 & 0.64 & 0.79 & 0.72 & 0.70 & 0.68 & 0.73 & 0.47 & 0.41 \\
\hline${ }^{230} \mathrm{Th}$ & & & & 0.36 & 0.18 & 0.16 & -0.19 & 0.58 & 0.03 & -0.22 & 0.42 & -0.05 & -0.15 & -0.44 & -0.39 & 0.21 & 0.53 & 0.74 & 0.66 & 0.63 & 0.62 & 0.73 & 0.32 & 0.17 \\
\hline${ }^{232} \mathrm{Th}$ & & & & & 0.13 & -0.23 & 0.04 & 0.27 & 0.09 & 0.44 & 0.05 & 0.27 & 0.06 & 0.25 & 0.27 & -0.03 & -0.02 & -0.12 & -0.19 & -0.15 & -0.17 & -0.05 & 0.07 & -0.58 \\
\hline $\mathrm{Cr}$ & & & & & & 0.52 & 0.65 & 0.81 & 0.22 & 0.62 & 0.90 & -0.03 & 0.77 & 0.53 & -0.55 & 0.03 & 0.22 & 0.05 & 0.04 & -0.01 & 0.02 & -0.22 & -0.03 & 0.20 \\
\hline $\mathrm{Pb}$ & & & & & & & 0.78 & 0.36 & 0.32 & 0.12 & 0.67 & -0.58 & 0.16 & -0.11 & -0.86 & -0.58 & 0.40 & 0.35 & 0.27 & 0.23 & 0.35 & 0.09 & -0.17 & 0.44 \\
\hline $\mathrm{Cu}$ & & & & & & & & 0.28 & 0.30 & 0.51 & 0.62 & -0.30 & 0.34 & 0.30 & -0.000 & -0.59 & 0.04 & -0.15 & -0.20 & -0.21 & -0.12 & $\frac{0.05}{-0.43}$ & $\begin{array}{l}-0.17 \\
-0.26\end{array}$ & 0.06 \\
\hline $\mathrm{Zn}$ & & & & & & & & & 0.38 & 0.39 & 0.86 & 0.09 & 0.51 & 0.30 & -0.44 & 0.26 & 0.04 & 0.40 & 0.36 & 0.30 & 0.30 & 0.25 & 0.20 & 0.25 \\
\hline Cd & & & & & & & & & & 0.29 & 0.00 & $\begin{array}{l}-0.09 \\
-0.12\end{array}$ & 0.27 & 0.06 & $\begin{array}{l}-0.44 \\
-0.03\end{array}$ & $\frac{0.20}{-0.02}$ & 0.49 & $\frac{0.40}{-0.05}$ & $\frac{0.00}{-0.09}$ & -0.15 & -0.15 & 0.25 & $\begin{array}{l}0.00 \\
-0.17\end{array}$ & 0.09 \\
\hline Co & & & & & & & & & & & 0.41 & 0.42 & $\begin{array}{l}0.27 \\
0.74\end{array}$ & 0.00 & $\begin{array}{l}-0.03 \\
0.14\end{array}$ & $\frac{-0.02}{-0.15}$ & $\frac{0.10}{-0.36}$ & $\begin{array}{l}-0.03 \\
-0.49\end{array}$ & $\begin{array}{l}-0.09 \\
-0.52\end{array}$ & $\frac{-0.13}{-0.53}$ & $\begin{array}{l}-0.13 \\
-0.49\end{array}$ & $\frac{0.00}{-0.56}$ & $\begin{array}{l}-0.17 \\
-0.41\end{array}$ & $\begin{array}{l}0.09 \\
-0.37\end{array}$ \\
\hline $\mathrm{Ni}$ & & & & & & & & & & & & $\frac{0.72}{-0.12}$ & 0.59 & $\frac{0.10}{0.20}$ & -0.69 & -0.01 & 0.37 & 0.31 & 0.27 & 0.21 & 0.23 & 0.08 & 0.05 & 0.33 \\
\hline Mn & & & & & & & & & & & & & 0.1 & 0.40 & $\begin{array}{l}0.059 \\
0.03\end{array}$ & 0.37 & $\begin{array}{l}-0.41 \\
\end{array}$ & $\frac{0.01}{-0.25}$ & $\frac{0.21}{-0.22}$ & -0.20 & $\frac{0.23}{-0.28}$ & $\frac{0.00}{-0.12}$ & $\begin{array}{l}0.02 \\
-0.02\end{array}$ & $\frac{0.39}{-0.39}$ \\
\hline $\mathrm{Fe}$ & & & & & & & & & & & & & & $\begin{array}{l}0.44 \\
0.67\end{array}$ & $\frac{0.05}{-0.05}$ & 0.14 & $\begin{array}{l}-0.41 \\
-0.21\end{array}$ & $\begin{array}{l}-0.25 \\
-0.29\end{array}$ & $\frac{-2.24}{-0.24}$ & $\begin{array}{l}-0.20 \\
-0.28\end{array}$ & $\frac{-0.20}{-0.30}$ & 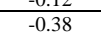 & $\begin{array}{l}-0.02 \\
-0.16\end{array}$ & 0.07 \\
\hline Al & & & & & & & & & & & & & & & 0.24 & 0.13 & -0.32 & -0.51 & -0.55 & -0.55 & -0.54 & -0.64 & -0.23 & -0.21 \\
\hline${ }^{206 / 207} \mathbf{P b}$ & & & & & & & & & & & & & & & & 0.24 & -0.50 & -0.48 & -0.42 & -0.37 & -0.46 & -0.17 & 0.01 & -0.53 \\
\hline${ }^{208 / 206} \mathbf{P b}$ & & & & & & & & & & & & & & & & & $\begin{array}{l}-0.03 \\
\end{array}$ & -0.06 & -0.01 & -0.05 & -0.16 & 0.07 & 0.11 & -0.11 \\
\hline LOI & & & & & & & & & & & & & & & & & & 0.82 & 0.81 & 0.79 & 0.83 & 0.67 & 0.60 & 0.40 \\
\hline${ }^{137} \mathrm{Cs}$ & & & & & & & & & & & & & & & & & & & 0.98 & 0.98 & 0.98 & 0.91 & 0.58 & 0.57 \\
\hline${ }^{239+240} \mathbf{P u}$ & & & & & & & & & & & & & & & & & & & & 0.99 & 0.98 & 0.90 & 0.69 & 0.60 \\
\hline${ }^{241} \mathbf{A m}$ & & & & & & & & & & & & & & & & & & & & & 0.98 & 0.90 & 0.73 & 0.57 \\
\hline${ }^{238} \mathrm{Pu}$ & & & & & & & & & & & & & & & & & & & & & & 0.87 & 0.63 & 0.58 \\
\hline Altitude & & & & & & & & & & & & & & & & & & & & & & & 0.57 & 0.40 \\
\hline Area & & & & & & & & & & & & & & & & & & & & & & & & 0.37 \\
\hline
\end{tabular}

Table S10. Results (H, p values) of Kruskal-Wallis test showing differences in heavy metals concentrations and radionuclide activity concentrations between cryoconites and soils. Statistically significant differences are bolded.

\begin{tabular}{|c|c|c|c|c|c|c|c|c|c|c|c|c|c|c|c|c|c|c|c|c|c|}
\hline & ${ }^{210} \mathrm{~Pb}$ & ${ }^{137} \mathrm{Cs}$ & ${ }^{239+240} \mathrm{Pu}$ & ${ }^{241} \mathrm{Am}$ & ${ }^{234} \mathrm{U}$ & ${ }^{238} \mathrm{U}$ & ${ }^{230} \mathrm{Th}$ & ${ }^{232} \mathrm{Th}$ & $\mathrm{Cr}$ & $\mathrm{Pb}$ & $\mathrm{Cu}$ & $\mathrm{Zn}$ & $\mathrm{Cd}$ & Co & $\mathrm{Ni}$ & $\mathrm{Mn}$ & $\mathrm{Fe}$ & $\mathrm{Al}$ & ${ }^{206 / 207} \mathrm{~Pb}$ & ${ }^{206 / 207} \mathrm{~Pb}$ & LOI \\
\hline $\mathrm{H}$ & 22.2 & 8.0 & 6.3 & 9.3 & 4.7 & 4.6 & 20.7 & 16.4 & 19.8 & 18.5 & 13.7 & 19.2 & 2.0 & $\begin{array}{ll}19.8 \\
\end{array}$ & 11.3 & 5.2 & 12.9 & 21.9 & 14.9 & 0.4 & 3.2 \\
\hline $\mathbf{p}$ & $\begin{array}{l}.00 \\
\end{array}$ & .00 & .01 & .00 & \begin{tabular}{|c|}
.03 \\
\end{tabular} & .03 & $\begin{array}{l}.00 \\
\end{array}$ & .00 & .00 & .00 & .00 & .00 & .16 & .00 & .00 & .02 & $\begin{array}{l}.00 \\
\end{array}$ & .00 & .00 & .05 & .07 \\
\hline
\end{tabular}

